\title{
Starlikeness of functions satisfying a differential inequality
}

\author{
by Rosihan M. Ali (Penang), \\ S. Ponnusamy (Madras) and Vikramaditya Singh (Kanpur)
}

\begin{abstract}
In a recent paper Fournier and Ruscheweyh established a theorem related to a certain functional. We extend their result differently, and then use it to obtain a precise upper bound on $\alpha$ so that for $f$ analytic in $|z|<1, f(0)=f^{\prime}(0)-1=0$ and satisfying $\operatorname{Re}\left\{z f^{\prime \prime}(z)\right\}>-\lambda$, the function $f$ is starlike.
\end{abstract}

1. Introduction and statement of results. Let $U$ be the unit disk $|z|<1$, and let $\mathcal{H}$ be the space of analytic functions in $U$ with the topology of local uniform convergence. The subclasses $A$ and $A_{0}$ of $\mathcal{H}$ consist of functions $f \in \mathcal{H}$ such that $f(0)=f^{\prime}(0)-1=0$ and $f(0)=1$ respectively. By $S, C, S t$ and $K$ we denote, respectively, the well known subsets of $A$ of univalent, close-to-convex, starlike (with respect to origin) and convex functions. Further, for $\beta<1$, we introduce

$$
\mathcal{P}_{\beta}=\left\{f \in A_{0}: \operatorname{Re} f(z)>\beta, z \in U\right\}
$$

and

$P_{\beta}=\left\{f \in A: \exists \alpha \in \mathbb{R}\right.$ such that $\left.\operatorname{Re}\left[e^{i \alpha}\left(f^{\prime}(z)-\beta\right)\right]>0, z \in U\right\}$.

If $f$ and $g$ are in $\mathcal{H}$ and have the power series

$$
f(z)=\sum_{k=0}^{\infty} a_{k} z^{k}, \quad g(z)=\sum_{k=0}^{\infty} \beta_{k} z^{k},
$$

the convolution or Hadamard product of $f$ and $g$ is defined by

$$
h(z)=(f * g)(z)=\sum_{k=0}^{\infty} a_{k} b_{k} z^{k} .
$$

1991 Mathematics Subject Classification: Primary 30C45.

Key words and phrases: univalent, convex, starlike, close-to-convex functions, duality of Hadamard products.

This work was carried out when the third author was visiting Universiti Sains Malaysia. 
For $V \subset A_{0}$ the dual $V^{*}$ of $V$ is the set of functions $g \in A_{0}$ such that $(f * g)(z) \neq 0$ for every $f \in V$, and $V^{* *}=\left(V^{*}\right)^{*}$.

We define functions $h_{T}$ in $A$ by

$$
h_{T}(z)=\frac{1}{1+i T}\left[i T \frac{z}{1-z}+\frac{z}{(1-z)^{2}}\right], \quad T \in \mathbb{R},
$$

and the subclass $V_{\beta}$ of $A_{0}$ by

$$
V_{\beta}=\left\{(1-\beta) \frac{1-x z}{1-y z}+\beta:|x| \leq 1,|y| \leq 1, \beta<1\right\} .
$$

We refer to $[2,3]$ for results in duality theory.

For a suitable $\Lambda:[0,1] \rightarrow \mathbb{R}$ define

$$
L_{\Lambda}(f)=\inf _{z \in U} \int_{0}^{1} \Lambda(t)\left[\operatorname{Re} \frac{f(t z)}{t z}-\frac{1}{(1+t)^{2}}\right] d t, \quad f \in C
$$

and

$$
L_{\Lambda}(C)=\inf _{f \in C} L_{\Lambda}(f)
$$

In a recent paper [1] Fournier and Ruscheweyh have established the following

ThEOREM A. Let $\Lambda$ be integrable on $[0,1]$ and positive on $(0,1)$. If $\Lambda(t) /\left(1-t^{2}\right)$ is decreasing on $(0,1)$ then $L_{\Lambda}(C)=0$.

The functions

$$
\Lambda_{c}(t)= \begin{cases}\left(1-t^{c}\right) / c, & -1<c \leq 2, c \neq 0 \\ \log (1 / t), & c=0\end{cases}
$$

satisfy the conditions of Theorem A.

It is clear that Theorem A can be extended to the case of $t \Lambda(t)$ integrable on $[0,1]$, positive on $(0,1)$, and $t \Lambda(t) /\left(1-t^{2}\right)$ decreasing on $(0,1)$. Indeed,

$$
\begin{aligned}
\int_{0}^{1} \Lambda(t) \operatorname{Re} & \left\{\frac{h_{T}(t z)}{t z}-\frac{1}{(1+t)^{2}}\right\} d t \\
& =\int_{0}^{1} t \Lambda(t) \operatorname{Re}\left\{\frac{1}{1+i T}\left[\frac{i T z}{1-t z}+\frac{z(2-t z)}{(1-t z)^{2}}\right]+\frac{2+t}{(1+t)^{2}}\right\} d t
\end{aligned}
$$

which shows that integrability of $t \Lambda(t)$ is enough for the existence of the integral. Further, if $t \Lambda(t) /\left(1-t^{2}\right)$ is decreasing, so is $\Lambda(t) /\left(1-t^{2}\right)$ and hence the treatment in [1] gives the result. Thus the functions

$$
\Lambda_{c}(t)=\left(1-t^{c}\right) / c, \quad-2<c \leq-1,
$$

satisfy the above conditions.

In the present paper we extend Theorem A in the following form. 
Theorem 1. For $\Lambda$ not integrable on $[0,1]$, let $t \Lambda(t)$ be integrable on $[0,1]$, positive on $(0,1)$, and suppose

$$
\Lambda(t) /\left(1-t^{2}\right) \text { is decreasing on }(0,1) \text {. }
$$

Then $L_{\Lambda}(C)=0$.

We use the theorem to establish the following:

Theorem 2. Suppose $\alpha:[0,1] \rightarrow \mathbb{R}$ is non-negative with $\int_{0}^{1} \alpha(t) d t=1$,

$$
\Lambda(t)=\int_{t}^{1} \frac{\alpha(t)}{t^{2}} d t
$$

satisfies the conditions of Theorem 1 and for $\lambda>0$, define

$$
V_{\alpha}(f)=z \int_{0}^{1}\left(1+\frac{\lambda z}{1-t z}\right) \alpha(t) d t * f(z), \quad f \in A .
$$

Then for $\lambda$ given by

$$
2 \lambda \int_{0}^{1} \frac{\alpha(t)}{1+t} d t=1
$$

we have $V_{\alpha}\left(P_{0}\right) \subset S$, and

$$
V_{\alpha}\left(P_{0}\right) \subset S t \Leftrightarrow L_{\Lambda}(C)=0 .
$$

For any larger value of $\lambda$ there exists an $f \in P_{0}$ with $V_{\alpha}(f)$ not even locally univalent.

As a special case of the above theorem we obtain a result which is interesting enough to be stated as a theorem.

Theorem 3. If $\lambda>0$ and $f \in A$ satisfies the differential inequality

$$
\operatorname{Re} z f^{\prime \prime}(z)>-\lambda
$$

then $f \in S t$ if

$$
0<\lambda \leq 1 / \log 4
$$

For any larger value of $\lambda$, a function $f \in A$ satisfying (3) need not even be locally univalent.

ThEOREM 4. Let $\alpha:[0,1] \rightarrow \mathbb{R}$ be non-negative with $\int_{0}^{1} \alpha(t) d t=1$ and suppose $\Lambda(t)=\alpha(t) / t$ satisfies the conditions of Theorem 1 . If $V_{\alpha}(f)$ is defined by $(1)$, then

and $\lambda$ is given by

$$
V_{\alpha}\left(P_{0}\right) \subset K \Leftrightarrow L_{\Lambda}(C)=0
$$

$$
2 \lambda \int_{0}^{1} \frac{2+t}{(1+t)^{2}} \alpha(t) d t=1
$$


2. Proof of Theorem 1. For a fixed $f \in C$ and $z \in U$ let

$$
\operatorname{tg}(t)=\operatorname{Re} \frac{f(t z)}{t z}-\frac{1}{(1+t)^{2}}
$$

Then $g$ is analytic in $t$. Let

$$
\Lambda_{n}(t)= \begin{cases}\Lambda(t), & 1 / n \leq t \leq 1 \\ \frac{\left(1-t^{2}\right) \Lambda(1 / n)}{1-1 / n^{2}}, & 0 \leq t \leq 1 / n\end{cases}
$$

From Theorem A we get

$$
0 \leq \frac{n^{2}}{n^{2}-1} \Lambda\left(\frac{1}{n}\right) \int_{0}^{1 / n}\left(1-t^{2}\right) t g(t) d t+\int_{1 / n}^{1} t \Lambda(t) g(t) d t=H_{n}+G_{n}
$$

Now

$$
\left|H_{n}\right| \leq \frac{\Lambda(1 / n)}{2\left(n^{2}-1\right)} M_{1} \rightarrow 0 \quad \text { as } n \rightarrow \infty .
$$

Let $\chi_{n}(t)$ be the characteristic function of $[1 / n, 1]$. For each $n$,

$$
\left|t \Lambda(t) g(t) \chi_{n}(t)\right| \leq M_{2} t \Lambda(t)
$$

Since $t \Lambda(t)$ is integrable, it follows that

$$
\lim _{n \rightarrow \infty} G_{n}=\lim _{n \rightarrow \infty} \int_{0}^{1} t \Lambda(t) g(t) \chi_{n}(t) d t=\int_{0}^{1} t \Lambda(t) g(t) d t .
$$

Hence $L_{\Lambda}(f) \geq 0$ for $z \in U$. This completes the proof.

We are thankful to Prof. S. Ruscheweyh for his help with the proof of Theorem 1.

3. Proof of Theorems 2 and 3. For $f \in P_{0}$ let $F(z)=V_{\alpha}(f)$. We then have

$$
F^{\prime}(z)=\int_{0}^{1}\left(1+\frac{\lambda z}{1-z t}\right) \alpha(t) d t * f^{\prime}(z), \quad f \in P_{0} .
$$

Since $V_{0}^{*}=\mathcal{P}_{1 / 2}$ and $V_{0}^{* *}=\left\{f^{\prime}: f \in P_{0}\right\}, F^{\prime}(z) \neq 0$ if and only if

$$
\frac{1}{2}<\operatorname{Re} \int_{0}^{1}\left(1+\frac{\lambda z}{1-z t}\right) \alpha(t) d t .
$$

This gives

$$
\lambda \int_{0}^{1} \frac{\alpha(t)}{1+t} d t \leq \frac{1}{2}
$$

Further, because $\operatorname{Re} e^{i \alpha} f^{\prime}(z)>0$, (5) also ensures that $\operatorname{Re} e^{i \alpha} F^{\prime}(z)>0$ and hence $F$ is univalent. 
For starlikeness we use the easily verifiable property that $F \in A$ is in $S t$ if and only if

$$
\frac{1}{z}\left(F * h_{T}\right)(z) \neq 0, \quad T \in \mathbb{R}, z \in U
$$

This gives

$$
\begin{aligned}
0 & \neq \int_{0}^{1}\left(1+\frac{\lambda z}{1-t z}\right) \alpha(t) d t * \frac{h_{T}(z)}{z} * \frac{f(z)}{z} \\
& =\int_{0}^{1}\left[1+\frac{\lambda}{t}\left\{\frac{1}{z} \int_{0}^{z}\left(\frac{h(t w)}{t w}-1\right) d w\right\}\right] \alpha(t) d t * f^{\prime}(z), \quad f \in P_{0} .
\end{aligned}
$$

This implies that $F \in S t$ if and only if

$$
\frac{1}{2}<\operatorname{Re} \int_{0}^{1}\left[1+\frac{\lambda}{t}\left\{\frac{1}{z} \int_{0}^{z}\left(\frac{h(t w)}{t w}-1\right) d w\right\}\right] \alpha(t) d t .
$$

On substituting the value of $\lambda$ from (2) in the above inequality, we obtain

$$
0<\operatorname{Re} \int_{0}^{1} \frac{\alpha(t)}{t^{2}}\left\{\frac{1}{z} \int_{0}^{z}\left(\frac{h(t w)}{w}-\frac{t}{1+t}\right) d w\right\} d t .
$$

This is similar to the last equation in [1]. Hence we need

$$
\Lambda(t)=\int_{t}^{1} \frac{\alpha(t)}{t^{2}} d t
$$

in order to use Theorem A. This completes the proof.

For the proof of Theorem 3 we take $\alpha(t) \equiv 1$. Then $\Lambda(t)=1 / t-1$ satisfies the conditions of Theorem 1 and $F$ satisfies (3). For $\alpha(t) \equiv 1$ the value of $\lambda$ obtained from (2) gives (4).

Notice that in (3), $\lambda=0$ only if $f(z) \equiv z$. Thus functions of the form

$$
\varrho z+(1-\varrho) f(z), \quad \varrho<1,
$$

where $f$ satisfies $(3)$, are in $S t$ for $(1-\varrho) \lambda \leq 1 / \log 4$.

Further, if $f \in A$ satisfies (3), then for a non-negative $\alpha$ satisfying $\int_{0}^{1} \alpha(t) d t=1$, the functions

$$
\phi(z)=\int_{0}^{1} \frac{\alpha(t)}{t} f(t z) d t
$$

also satisfy (3) and hence are starlike for the same value of $\lambda$. 
4. Proof of Theorem 4. We need to prove that $z F^{\prime}(z) \in S t, F(z)=$ $V_{\alpha}(f)$. Hence $(6)$ gives

$$
\begin{aligned}
0 & \neq F^{\prime}(z) * \frac{h_{T}(z)}{z} \\
& =\int_{0}^{1}\left(1+\frac{\lambda z}{1-t z}\right) \alpha(t) d t * \frac{h_{T}(z)}{z} * f^{\prime}(z) \\
& =\int_{0}^{1}\left[1+\frac{\lambda}{t}\left(\frac{h(t z)}{t z}-1\right)\right] \alpha(t) d t * f^{\prime}(z), \quad f \in P_{0} .
\end{aligned}
$$

This holds if and only if

$$
\frac{1}{2}<\operatorname{Re} \int_{0}^{1}\left[1+\frac{\lambda}{t}\left(\frac{h(t z)}{t z}-1\right)\right] \alpha(t) d t .
$$

Substitution of the value of $\lambda$ in the theorem gives

$$
0<\operatorname{Re} \int_{0}^{1}\left[\frac{h(t z)}{t z}-\frac{1}{(1+t)^{2}}\right] \frac{\alpha(t)}{t} d t .
$$

Hence with $\Lambda(t)=\alpha(t) / t$, Theorem 1 gives the result.

The choice of $\alpha(t)=2(1-t)$ gives the result of Theorem 3 with $\lambda$ replaced by $2 \lambda$.

\section{References}

[1] R. Fournier and S. Ruscheweyh, On two extremal problems related to univalent functions, Rocky Mountain J. Math. 24 (1994), 529-538.

[2] S. Ruscheweyh, Duality for Hadamard products with applications to extremal problems for functions regular in the unit disc, Trans. Amer. Math. Soc. 210 (1975), 63-74.

[3] - Convolution in Geometric Function Theory, Les Presses de l'Université de Montréal, Montréal, 1982.

SCHOOL OF MATHEMATICAL AND COMPUTER SCIENCES UNIVERSITI SAINS MALAYSIA 11800 PENANG, MALAYSIA

SCHOOL OF MATHEMATICS SPIC SCIENCE FOUNDATION 92 G.N. CHETTY ROAD MADRAS 600 017, INDIA
3A/95 AZAD NAGAR KANPUR 208002, INDIA 